\title{
Numerical damage identification of structures by observability techniques based on static loading tests
}

\begin{abstract}
This paper proposes the application of the observability techniques to deal with damage detection in bridges from their structural response under static loading tests. Unlike previous works based on a symbolic approach to this technique, this paper presents its first numerical application. With this aim, a novel algorithm is presented, which reduces the unavoidable numerical errors produced by the lack of precision of computers. To achieve an adequate accuracy in estimations, this numerical algorithm is complemented with another method to define the proper geometry of the corresponding finite element model. The comparison of the observability technique with other existing methods presented in the literature shows that the number of required measurements is significantly lower. Furthermore, contrary to other analyzed methods, no information from the undamaged structure is required. The accuracy in estimations provided by the proposed method is very high as the differences with actual values are lower than $1 \%$.
\end{abstract}

KEYWORDS: Non-destructive testing, Damage detection, Bridge deck, Static test, Structural response, Numerical analysis, Precision error.

\section{INTRODUCTION}

Structural-System Identification (SSI) can be used to identify damage in actual structures based on their structural response on site (see ASCE (2001)). An adequate damage detection method must be able to locate and quantify damage in actual structures. Once identified, damage can be modeled in the structural Finite Element Model (FEM), increasing its accuracy and helping the decision making during maintenance [see (Castillo et al. 2014)].

Damage might be detected by visual inspections. These inspections can be complemented with other nondestructive techniques. Nevertheless, these two methods might be insufficient in complex structures where damage is invisible to human eyesight or the members are not accessible. In these cases damage can be detected by non-destructive tests that measure the structural response to a certain excitation. According to the type of 
excitation, these tests can be classified as dynamic [Zhang et al. (2014), Chao and Loh (2014) or Chang et al. (2014)] or static. Static methods are usually simpler than dynamic ones. For this reason, the static-excitation based SSI methods have attracted much attention from the 90s [Banan and Hjelmstad (2003)]. Sanayei and Scamboli (1991) estimated structural stiffnesses by minimizing the static-stiffness based error function. In this work, an iterative identification procedure was used to automatically adjust the elemental stiffness parameters for damage detection. Sanayei and Onipede (1991) presented an iterative optimization-based algorithm of the displacement equation error function for the parameter identification based on static test measurements. To deal with incomplete measurements, a condensation procedure was proposed. This method was used by Sanayei et al. (1992) to determine the effects of measurement errors. Hjelmstad et al. (1992) described an approach for parameter estimation of complex linear structures based on the principle of virtual work for static and modal tests. Banan et al. (1994a, b) proposed an optimization method to estimate member constitutive properties of the finite element model from measured displacements under static loading. This analysis was based on the minimization of two discrepancy indices between model and measurements using the constrained least-square minimization. Sanayei and Saletnik (1996a) developed an iterative method for parameter estimation by minimizing an error function in truss and framed structures. A sensitivity analysis of the measurements errors of this method was presented in Sanayei and Saletnik (1996b). Hjemstad and Shin (1997), proposed an adaptive parameter algorithm to detect and to assess damage in a structural system. Liu and Chian (1997) developed a method to identify truss properties using axial strains by minimizing the error norm of the equilibrium equation. Recently, static damage detection methods are receiving attention again from researchers. Bakhtiari-Nejad et al. (2005) introduced a damage detection method using static noisy measurements in which the difference between the load vector based on damaged and undamaged structure was minimized. Sanayei et al. (2012) used measured strains in a real bridge under static truck loads for FEM updating. Liao et al. (2012) presented a FEM updating method that included an iterative process based on the influence line of the damaged structure. Abdo (2012) used the changes in the displacement curvature derived from measured static response to locate damage in beams. Viola and Bocchini (2013) proposed the use of genetic algorithms for parametric damage detection in 
truss structures. Recently, Rezaiee-Pajand et al. (2014) proposed the minimization of differences between measured and analytical static displacements of frames for damage detection. In this approach a nonlinear constrained structural optimization based on the eigen-decomposition of the local elemental stiffness matrix was suggested. Lozano-Galant et al. $(2013,2014,2015)$ proposed the application of observability techniques to structural system identification from a symbolic point of view. The advantage of using this approach is that the mathematical foundation of the method can be completely understood and checked. Nevertheless, for the method to be implemented practically, a numerical analysis is required. To fill this gap, this paper presents the first numerical application of the observability technique. This application includes the development of an algorithm that reduces the unavoidable numerical errors produced by the lack of precision of computers. This algorithm is complemented with another that addresses the efficient definition of the finite element model. To validate the proposed technique, the obtained results are compared with those alternative methods recently presented in the literature.

This paper is based on the numerical development of the observability techniques. For the sake of convenience the main assumptions of the observability method presented in the literature are first described in Section 2. In Section 3, the observability techniques are adapted to deal with the adequate numerical damage detection of structures. With this aim, an algorithm that solves the numerical problems of the observability technique is presented for the first time in the literature. This algorithm is named as Numerical Observability Method (NOM). This section also includes the definition of another algorithm to address the efficient definition of the finite element model for damage detection. In Section 4, the damage detection method by observability techniques is numerically compared with two alternative methods recently presented in the literature. Finally, some conclusions are drawn in Section 5.

\section{STRUCTURAL SYSTEM IDENTIFICATION BY OBSERVABILITY TECHNIQUES}

In the stiffness matrix method, the equilibrium equations together with strength of materials theory might be written in terms of node displacements and node forces as presented in Eq. 1. 
$[\mathrm{K}] \cdot\{\delta\}=\{\mathrm{f}\}$

in which $[\mathrm{K}]$ is the stiffness matrix of the structure, $\{\delta\}$, is a vector of node displacements and $\{\mathrm{f}\}$ is a vector of node forces. For 2D analysis, Matrix $[\mathrm{K}]$ includes the geometrical and mechanical properties of the beam elements of the structure, such as length, $\mathrm{L}_{\mathrm{j}}$, shear modulus, $\mathrm{G}_{\mathrm{j}}$, Young's modulus, $\mathrm{E}_{\mathrm{j}}$, area, $\mathrm{A}_{\mathrm{j}}$, inertia, $\mathrm{I}_{\mathrm{j}}$, and torsional stiffness, $\mathrm{J}_{\mathrm{j}}$, associated with the $\mathrm{j}$-element. For the sake of simplicity, in this paper the structural mechanisms involved are limited to the axial stiffness, $E_{j} A_{j}$, and the flexural stiffness, $E_{j} I_{j}$.

When the SSI is introduced in the stiffness matrix method, the matrix $[\mathrm{K}]$ is partially unknown. Usually, $\mathrm{L}_{\mathrm{j}}$ is assumed known while both the flexural, $E_{j} I_{j}$, and axial stiffness, $E_{j} A_{j}$, are traditionally assumed as unknown. As there could also be uncertainties in actual areas of the members of the structure and/or in densities, the applied forces in nodes due to permanent loads might also be unknown. This problem can be overcome by analyzing the effects of the increment of deflections produced by given sets of nondestructive static tests.

The determination of the unknown parameters in matrix $[\mathrm{K}]$ leads to a nonlinear problem as these parameters are multiplied by the displacements of the nodes (in $2 \mathrm{D}$, horizontal and vertical deflection and rotation $\mathrm{u}_{\mathrm{k}}, \mathrm{v}_{\mathrm{k}}$, $\mathrm{w}_{\mathrm{k}}$ associated with the k-node). This implies that non-linear products of variables, such as $\mathrm{E}_{\mathrm{j}} \mathrm{A}_{\mathrm{j}} \mathrm{u}_{\mathrm{k}}, \mathrm{E}_{\mathrm{j}} \mathrm{A}_{\mathrm{j}} \mathrm{v}_{\mathrm{k}}$, $\mathrm{E}_{\mathrm{j}} \mathrm{I}_{\mathrm{j}} \mathrm{u}_{\mathrm{k}}, \mathrm{E}_{\mathrm{j}} \mathrm{I}_{\mathrm{j}} \mathrm{v}_{\mathrm{k}}$ and $\mathrm{E}_{\mathrm{j}} \mathrm{I}_{\mathrm{j}} \mathrm{w}_{\mathrm{k}}$, might appear, leading to a polynomial system of equations. To solve these equations in a linear-form, system (1) can be rewritten as

$\left[\mathrm{K}^{*}\right] \cdot\left\{\delta^{*}\right\}=\{\mathrm{f}\}$,

in which the products of variables are located in the modified vector of displacements $\left\{\delta^{*}\right\}$ and the modified stiffness matrix $\left[\mathrm{K}^{*}\right]$ is a matrix of coefficients with different dimensions than the initial stiffness matrix $[\mathrm{K}]$. Depending on the known information, the unknown variables of vector $\left\{\delta^{*}\right\}$ may be the non-linear products presented above, as well as other factors of single variables, such as $E_{j} I_{j}, E_{j} A_{j}, E_{j}, A_{j}, I_{j}$ or node deflections.

Once the boundary conditions and the applied forces at the nodes during the nondestructive test are introduced, it can be assumed that a subset of increments of deflections $\delta_{1}^{*}$ of $\left\{\delta^{*}\right\}$ and a subset of forces in 
nodes $\mathrm{f}_{1}$ of $\{\mathrm{f}\}$ are known and the remaining subset $\delta_{0}^{*}$ of $\left\{\delta^{*}\right\}$ and $\mathrm{f}_{0}$ of $\{\mathrm{f}\}$ are not. By the static condensation procedure, the system in (2) can be partitioned as follows:

$\left[\mathrm{K}^{*}\right]\left\{\delta^{*}\right\}=\left(\begin{array}{ll}\mathrm{K}_{00}^{*} & \mathrm{~K}_{01}^{*} \\ \mathrm{~K}_{10}^{*} & \mathrm{~K}_{11}^{*}\end{array}\right)\left\{\begin{array}{l}\delta_{0}^{*} \\ \delta_{1}^{*}\end{array}\right\}=\left\{\begin{array}{l}\mathrm{f}_{0} \\ \mathrm{f}_{1}\end{array}\right\}=\{\mathrm{f}\}$

where $\mathrm{K}_{00}^{*}, \mathrm{~K}_{01}^{*}, \mathrm{~K}_{10}^{*}$ and $\mathrm{K}_{11}^{*}$ are partitioned matrices of $\left[K^{*}\right]$ and $\delta_{0}^{*}, \delta_{1}^{*}, \mathrm{f}_{0}$ and $\mathrm{f}_{1}$ are partitioned vectors of $\left\{\delta^{*}\right\}$ and $\{f\}$.

In order to join the unknowns, system (3) can be written in the equivalent form, as:

$$
[B]\{z\}=\left(\begin{array}{cc}
K_{10}^{*} & 0 \\
K_{00}^{*} & -\mathrm{I}
\end{array}\right)\left\{\begin{array}{c}
\delta_{0}^{*} \\
\mathrm{f}_{0}
\end{array}\right\}=\left\{\begin{array}{l}
\mathrm{f}_{1}-K_{11}^{*} \times \delta_{1}^{*} \\
-K_{01}^{*} \times \delta_{1}^{*}
\end{array}\right\}=\{D\}
$$

where 0 and $I$ are the null and the identity matrices, respectively. In this system the vector of unknown variables, $\{z\}$, appears on the left-hand side and the vector of observations, $\{\mathrm{D}\}$, on the right-hand side. Both vectors are related by a coefficient matrix[B].

Matrix $\{D\}$ must satisfy some conditions for the system (4) to have a solution. In order to check if the system has a solution it is sufficient to calculate the null space $[\mathrm{V}]$ of $[\mathrm{B}]$ and checking that $[\mathrm{V}][\mathrm{D}]=\{0\}$. The general solution (the set of all solutions) of the system (4) has the structure:

$$
\left\{\begin{array}{l}
\delta_{0}^{*} \\
f_{0}
\end{array}\right\}=\left\{\begin{array}{c}
\delta_{00}^{*} \\
f_{00}
\end{array}\right\}+[V]\{\rho\}
$$

where $\left\{\begin{array}{c}\delta_{00}^{*} \\ f_{00}\end{array}\right\}$ is a particular solution of system (5) and $[\mathrm{V}]\{\rho\}$ is the set of all solutions of the associated homogeneous system of equations (a linear space of solutions, where the columns of [V] is a basis and the elements of the column matrix $\{\rho\}$ are arbitrary real values which represent the coefficients of all possible linear combinations).

Examination of matrix [V] and identification of its null rows leads to identification of the observable variables (subset of variables with a unique solution) of vector $\{\mathrm{z}\}$. To obtain matrix $[\mathrm{V}]$ we need to calculate the null space of matrix [B]. The value of the observed parameters (particular solution in (5)) can be obtained 
numerically by solving system (4).

The unknown parameters of vector $\{z\}$ can be directly identified from the null space when enough information is introduced into the observability analysis. Nevertheless, the number of required deflections can be optimized by using a recursive process that takes advantage of the connectivity of the beams in the stiffness matrix. This connectivity is included in partitioned matrices of $\left[K^{*}\right]$ and therefore, in system (4). In this way, when in the initial observability analysis any deflection, force or structural parameter is observed, this information might help to observe new parameters in the adjacent beams through a recursive process. In this analysis, the observed information is successively introduced as input data in the observability analysis.

A detailed step by step application of the observability techniques is presented in Lozano-Galant et al. (2013, 2014). We refer the reader to those papers for a more detailed explanation of the peculiarities of the proposed methodology.

\section{APPLICATION OF OBSERVABILITY TECHNIQUES TO NUMERICAL DAMAGE DETECTION}

The symbolical SSI algorithm presented in the preceding section fails to address the numerical estimation of the observed parameters. To solve this problem, the numerical development of the observability techniques is presented in this section by means of a new algorithm called Numerical Observability Method (NOM). As the precision of the estimations depends, to a great extent, on the geometry of the finite element models, this section also includes some guidelines to address the efficient definition of the finite element models assumed for damage detection.

\subsection{The Numerical Observability Method}

It is noted that the numerical application of the observability technique is not a straightforward task because of the errors in measurements and the lack of precision in computers and numerical procedures. In fact, the direct numerical application of the procedure presented in the preceding section might lead to the following 
problems:

- (1) Lack of observability during the recursive process. In each iteration, the observable variables of $\{\mathrm{z}\}$ are obtained from the analysis of the null space [V]. This is to say, the variables in $\{z\}$ are identified as observable when their corresponding rows in [V] are only composed only of zeros. This identification can be easily carried out symbolically. Nevertheless, because of the unavoidable numerical errors, this might not be the case when the structure is analyzed numerically. In such a case, next to zero values might appear in [V] instead of null values. This fact reduces the number of observed parameters in each step.

- (2) Numerical problems associated with the resolution of the system of equations. The symbolic analysis presented in the preceding section is limited to the identification of the list of parameters that are mathematically observable when a set of measurements are known. Nevertheless, in the numerical analysis it is necessary to go one step further as the values of the observable parameters must also be calculated. For this purpose, the system presented in Equation 5 needs to be solved. If the system has a unique solution, this can be easily solved by using the inverse of [B]. Nevertheless, this is rarely the case because the number of unknown variables in $\{z\}$ generally exceeds the number of equations. Therefore, alternative numerical methods are required. Examples of these methods are the LU factorization [Remon et al. (2006)], the MoorePenrose pseudo inverse [Yang and Zhang (2014)], or the least-squares method. Nevertheless, these methods can fail to provide the numerical solution because the actual numerical data generally contain inaccuracies leading to singular or nearly singular matrices.

- (3) The errors might increase throughout the recursive process. Due to the fact that numerical errors are unavoidable, the estimated parameters will not be free of errors. Furthermore, these errors might increase throughout the recursive process as the new parameters obtained in later steps are calculated from a higher number of parameters with errors. It is important to highlight that if this procedure is not controlled, accumulated errors at the end of the recursive process might lead to unacceptable estimations. This problem discourages the use of a purely numerical analysis approach. 
To solve all these problems a new algorithm is proposed. This algorithm combines two approaches: a symbolical and a numerical one. On the one hand, the symbolic approach is used for the observability analysis. This approach reduces the effects of the unavoidable numerical errors. The symbolic analysis solves two of the problems presented above (the number of observed parameters is not reduced and the errors in estimations do not increase during the recursive steps). On the other hand, the numerical approach enables the numerical estimation of the observed parameters. This mixed algorithm also includes a recursive process, in which the new observed parameters are successively introduced symbolically. The different steps of the proposed algorithm are presented in Fig. 1 and summarized as follows:

- INPUT: The topology of the structure and the subset $\mathrm{M}$ of measured (known) variables.

- OUTPUT: The numerical values of the resulting subset of observable variables O.

- Step 1: The initial input data are introduced. These data include the geometry of the FEM, the known mechanical and geometrical properties of the elements, the boundary conditions and the value of the deflection and force increments at the nodes during the static load test.

- Step 2: The partitioned matrices $\mathrm{K}_{00}^{*}, \mathrm{~K}_{01}^{*}, \mathrm{~K}_{10}^{*}$ and $\mathrm{K}_{11}^{*}$ are symbolically determined from input data.

- Step 3: The coefficient matrix [B], vector of observations, $\{\mathrm{D}\}$, and vector of unknown variables $\{\mathrm{z}\}$ are symbolically determined as presented in Eq. 4.

- Step 4: The $\mathrm{N}_{\mathrm{i}}$ parameters of vector $\{\mathrm{z}\}$ whose value can be unequivocally defined (this is to say, that are observable) are determined by analyzing the symbolic null space, [V], of matrix [B].

- Step 5: The numerical values of the observable parameters of $\{z\}$ are obtained from the particular solution of the system $[B] \cdot\{z\}=\{D\}$. This step is carried out by using an algorithm that provides the numerical solution of the system of equations. Examples of this kind of algorithms are the MoorePenrose pseudo inverse or the Least-Squares method.

- Step 6: If some parameters are observed, $\left(\mathrm{N}_{\mathrm{i}} \neq 0\right)$, the recursive process (with $\mathrm{i}$ counter) is carried out to update the input data of the observability analysis. In this way, the symbolic representation of the observed parameters is introduced as input data in a new observability analysis that corresponds with the 
following step of the recursive process $(\mathrm{i}=\mathrm{i}+1)$. To avoid the effects of the numerical errors, this new analysis is carried out symbolically.

- Step 7: The recursive process is repeated until no additional variables are observed between two successive steps $\left(\mathrm{N}_{\mathrm{i}}=\mathrm{N}_{\mathrm{i}-1}\right)$.

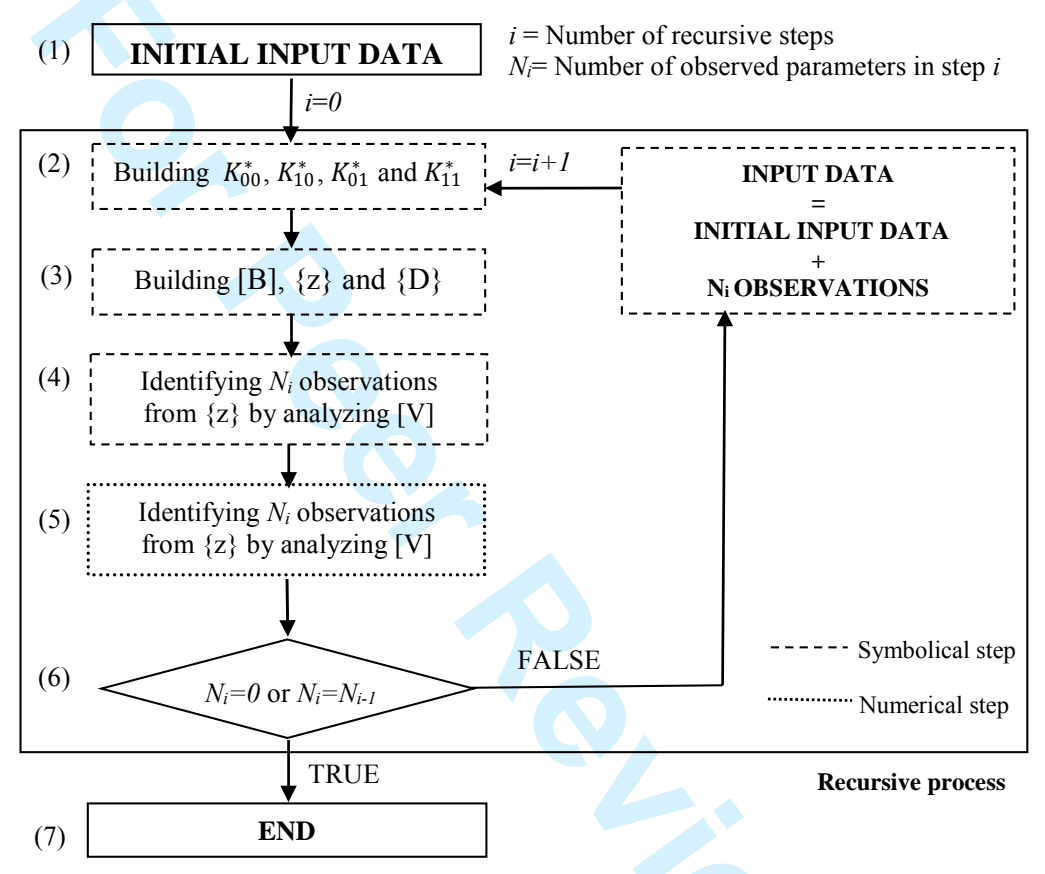

Fig. 1: Flow chart for the numerical solution of the SSI problem by observability techniques.

The accuracy of the estimations depends to a great extent on the assumptions of the finite element model. In fact, the higher the number of unknowns of this model the higher the number of measurements required for identification and the higher the accuracy. This issue is addressed in the following subsection.

\subsection{Defining an adequate FEM}

The number of beam elements and nodes of the Finite Element Model (FEM) influences both the number of information required in the structural system identification and the accuracy of the estimations. Obviously, the more complex the model, the higher the required information and the corresponding accuracy in estimations are.

As the damage location is not generally known, the following algorithm is proposed for the effective definition of the FEM. Coupling this algorithm with the NOM, damage can be estimated with a given tolerance. 


\section{1}

With the aim of understanding the advantages of this algorithm, the following aspects have to be taken into account: (1) Number of beam elements: The number of beam elements will depend on the number of different mechanical and geometrical properties as well as the assumed length of the damage extent. A different beam element (with unknown mechanical properties) will be defined in every cross section whose mechanical properties are to be estimated. Obviously, the higher the number of beam elements with unknown mechanical properties, the higher the amount of required information to be measured from the static test. (2) Number of nodes. A node is introduced at both edges of the different beam elements. Additional intermediate nodes can be introduced to avoid the need of measuring node rotations. (3) Balanced damage detection method. An adequate FEM for damage detection must conveniently balance the number of beam elements and their location, the number of nodes and their location and the pursued accuracy.

The proposed algorithm for an automatic definition of adequate FEM is as follows:

- Input data: Initial FEM, deflections or rotations measured under a certain load case and assumed tolerance in estimations.

- Output data: Estimated parameters fulfilling the aimed tolerance.

- Step 1: Definition of an initial FEM. Based on the knowledge of the structure and the designer's experience, an initial FEM is proposed.

- Step 2: Evaluation of the damages in the FEM: The damages of the FEM $\left(\mathrm{FEM}_{\mathrm{i}}\right)$ are evaluated numerically by the NOM.

- Step 3: Checking the obtained damages in consecutive beam elements: The differences between the parameters estimated by consecutive beam elements are analyzed. When these differences are higher than the assumed tolerance, this is to say, (Estimated parameter in element $\mathrm{j}+1$ - Estimated parameter in element j) > Tolerance, go to Step 4. Otherwise go to Step 5 .

- Step 4: Updating the geometry of the FEM: Those beam elements that in the preceding step exceeded the tolerance are divided into two, updating the geometry of the $\mathrm{FEM}_{\mathrm{i}}$. This division increases the number of 
nodes and beams and therefore, the number of unknown variables. After saving the deflections measured on site at the new node, go to Step 2 for a new SSI.

- Step 5: Merging beam elements with the same properties: If the geometry of two consecutive elements of the $\mathrm{FEM}_{\mathrm{i}}$ are equal, these two elements are merged into one.

- Step 6: The algorithm is finished and the estimated parameters are provided with an adequate tolerance between elements.

\section{COMPARISON WITH OTHER STATIC DAMAGE DETECTION METHODS}

In this section the observability technique is compared with two alternative damage detection methods presented in the literature. With this aim, the main assumptions of each of these methods are first presented. Then, to validate the proposed observability method, two examples presented in the literature are studied by observability techniques. Finally, the different damage detection methods are compared. The structural response of the developed FEMs is analyzed with the software SAP2000 neglecting the shear deformation.

\subsection{Method 1 (Liao et al. (2012))}

Liao et al. (2012) proposed a method for Finite Element Model updating based on the response of the structure under pseudo-static excitation test. In this method the magnitude of localized damage in beam elements are estimated by comparison of the influence lines of undamaged and damaged structures. In this method the influence lines of a moving load are determined by measuring the vertical deflections at a certain point. Liao et al.'s method compares the measured and the calculated influence lines by means of an objective function. In this way, the model updating problem is transformed into a classical constrained optimization problem. This problem is solved iteratively by the unconstrained minimization technique and Powell's method. One of the main disadvantages of this method is that it does not provide information about the damage location.

\subsubsection{Example 1 [Liao et al. (2012)]}

This example is based on the results of the experimental test program of the three beams presented in Fig. 2 
analyzed by Liao et al. (2012). All these beams are clamped at both ends and differences between them refer to their mechanical properties. The first beam (undamaged beam) presents a constant rectangular undamaged cross section UN, $15 \mathrm{~mm}$ width and $45 \mathrm{~mm}$ depth, as presented in Fig. 2.A. The other two beams (damaged beam 1 in Fig. 2.B and damaged beam 2 in Fig. 2.C) include damage at different locations. This damage is simulated by reducing the UN cross section by $15 \%$ (cross section $15 \times 38.25 \mathrm{~mm}^{2}$ ) and by $30 \%$ (cross section $15 \times 31 \mathrm{~mm}^{2}$ ) extended along $6 \mathrm{~cm}$. These cross sections (named 15\%D and 30\%D) are presented in Fig. 3.D together with UN. The Young's modulus are $2,67 \times 10^{3} \mathrm{MPa}$. It is assumed that damage only produces changes in the flexural stiffness. This assumption is traditionally used in many SSI methods (see Abdo (2012)).

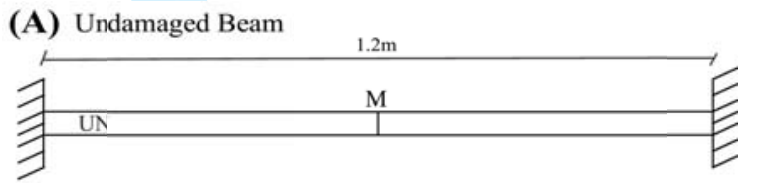

(B) Damaged Beam 1

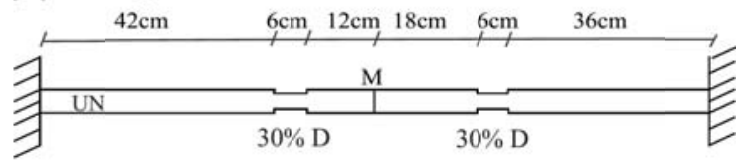

(C) Damaged Beam 2

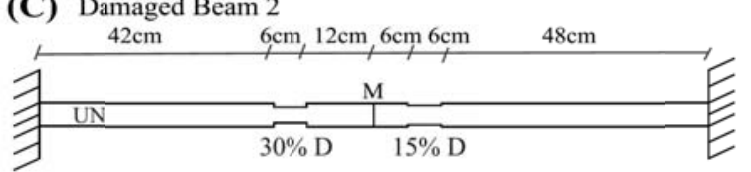

(D) Geometry $(\mathrm{cm})$

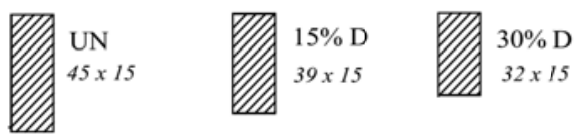

Fig. 2: Example 1 analyzed by Liao et al. (2012): (A) Undamaged beam. (B) Damaged Beam 1. (C) Damaged Beam 2. (D) Geometry. Undamaged section is named UN, sections $30 \% \mathrm{D}$ and $15 \% \mathrm{D}$ represents a damaged of $30 \%$ and $15 \%$, respectively.

Following the assumptions of the existing method, the damage location is known but not its magnitude. This results in a number of 5 unknown flexural stiffnesses, EI, named from $\mathrm{EI}_{1}$ to $\mathrm{EI}_{5}$. To define these values, the influence lines of the different damaged beams are compared with the one of the undamaged structure. In this case, the comparison is based on the vertical deflection influence lines at mid span (point $\mathrm{M}$ in Fig. 2.A, 2.B and 
2.C). These deflections are obtained by measuring the deflection at M produced by a moving concentrated load every $6 \mathrm{~cm}$. This results in a number of 19 measurements for each influence line. The maximum error in the estimated stiffnesses in the Damaged Beam 1 was $1.10 \%$. This value increases up to 1.94\% in Beam 2.

\subsubsection{Example 1 by observability techniques}

Estimation of the damaged magnitude of the two damaged structures presented in Example 1 can be carried out numerically by observability techniques. The first step consists of defining an adequate FEM to characterize conveniently the unknown mechanical properties. Using the procedure presented in Section 3.2 , two different FEMs can be defined for each damaged structure. These FEMs have the same number of nodes and beams (eight and seven, respectively) but different number of unknown stiffnesses. On the one hand, the first type of these models (FEM-1.1 in Fig. 3.A and FEM-2.1 in Fig. 3.B) assumes that all the undamaged elements have different stiffness properties. This assumption clusters the seven beams of the model in five unknown stiffnesses $\left(\mathrm{EI}_{1}\right.$ to $\left.\mathrm{EI}_{5}\right)$. On the other hand, the second type of models (FEM-1.2 in Fig. 3.C and FEM-2.2 in Fig. 3.D) assumes that the stiffnesses of the undamaged elements $\left(\mathrm{EI}_{1}, \mathrm{EI}_{3}\right.$ and $\left.\mathrm{EI}_{5}\right)$ are the same. This reduces the number of unknown stiffnesses to three. In both types of FEMs the flexural stiffnesses of the beam elements $\left(\mathrm{EI}_{1}\right.$ to $\mathrm{EI}_{5}$ in FEMs-1.1 and 2.1, $\mathrm{EI}_{1}$ to $\mathrm{EI}_{3}$ in FEMs-1.2 and FEMs-2.2) are unknown. For a certain load case, the forces in all FEM nodes except at the boundary conditions are assumed as known.

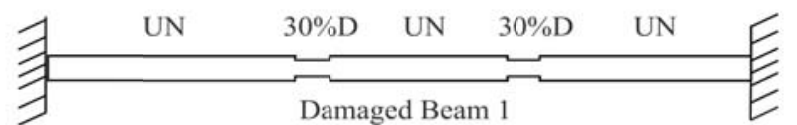

(A) FEM-1.1

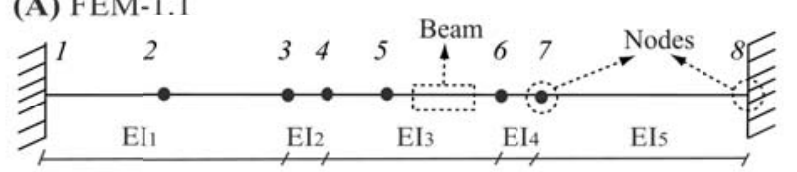

(C) FEM-1.2

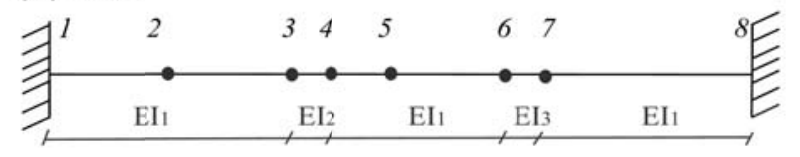

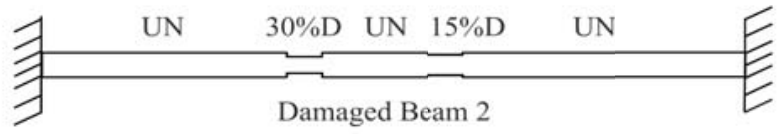

(B) FEM-2.1

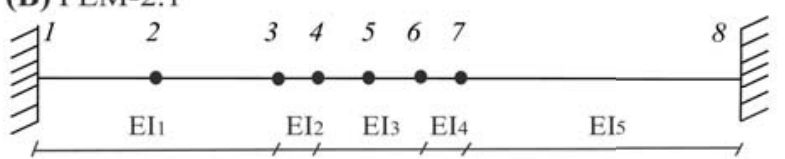

(D) FEM-2.2

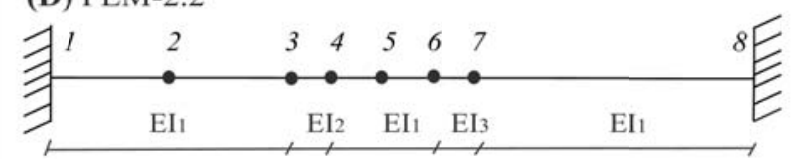

Fig. 3: Finite element models used by observability techniques in Example 1. (A) FEM-1.1, (B) FEM-2.1, (C) FEM-1.2 and (D) FEM-2.2. 
The size of the required measurement set for SSI depends on the number of unknown stiffnesses in the FEMs. Obviously, the higher this number, the higher the number of required measurements. In the case of FEM1.2 and 2.2, four rotations $\left(\mathrm{w}_{2}, \mathrm{w}_{3}, \mathrm{w}_{6}\right.$ and $\left.\mathrm{w}_{7}\right)$ are required while in FEM-1.1 and 2.1 this number increases up to six $\left(\mathrm{w}_{2}, \mathrm{w}_{3}, \mathrm{w}_{5}, \mathrm{w}_{6}, \mathrm{w}_{7}\right.$ and $\left.\mathrm{w}_{8}\right)$. The values of these parameters can be obtained from the increments of nodal rotations in a static test. In this example this test is considered as the application of a concentrated load Q of $20000 \mathrm{kN}$ at mid span (node 5). The increments of rotations obtained in this test are summarized in Table 1.

Table 1: Increments of nodal rotations in Example 1 when a concentrated load $\mathrm{Q}=20000 \mathrm{kN}$ is introduced at node 5 .

\begin{tabular}{|c|c|c|}
\hline & $\begin{array}{l}\text { Damaged beam } 1 \\
\text { (FEM } 1.1 \text { and } 1.2 \text { ) }\end{array}$ & $\begin{array}{l}\text { Damaged beam } 2 \\
\text { (FEM } 2.1 \text { and 2.2) }\end{array}$ \\
\hline $\mathrm{w}_{2}$ & -0.016037 & -0.016205 \\
\hline $\mathrm{w}_{3}$ & -0.016524 & -0.016729 \\
\hline $\mathrm{W}_{5}$ & -0.008216 & -0.013042 \\
\hline $\mathrm{w}_{6}$ & 0.001023 & -0.008376 \\
\hline $\mathrm{w}_{7}$ & 0.013023 & 0.000926 \\
\hline $\mathrm{W}_{8}$ & 0.017555 & 0.006233 \\
\hline & $\longrightarrow$ & EE \\
\hline
\end{tabular}

The results of the SSI are illustrated in Fig. 4. This figure includes the deviations between the original stiffnesses and the ones estimated by the different FEMs in Damaged Beam 1 (Fig. 4.A) and Damaged Beam 2 (Fig. 4. B). 


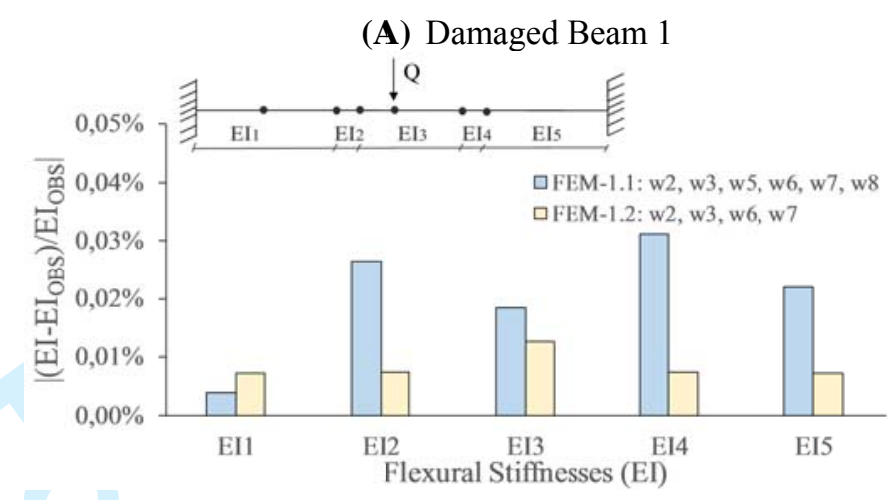

(B) Damaged Beam

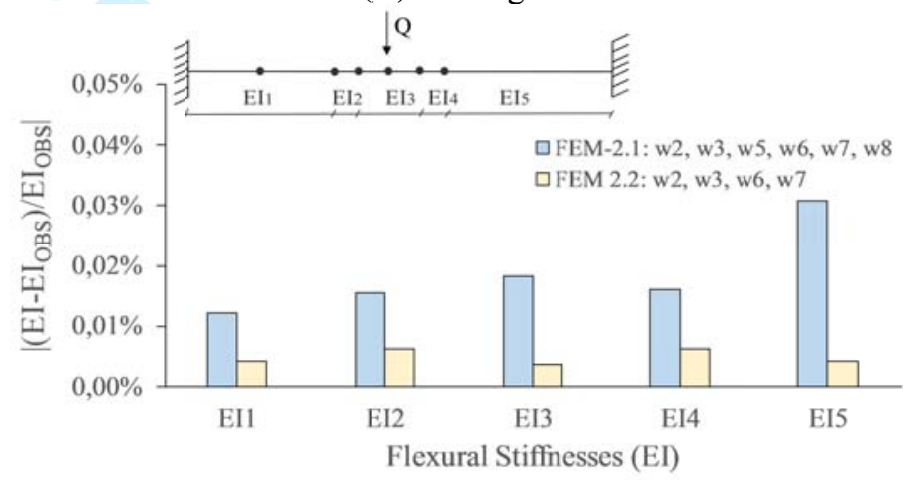

Fig 4: Example 1: Absolute value of the percentage deviations between the actual and the observed stiffnesses in different FEMs when a load $\mathrm{Q}=20000 \mathrm{kN}$ is applied at note 5. (A) Damaged Beam 1, (B) Damaged Beam 2.

Fig. 4.A and 4.B illustrate the accuracy of the five estimated stiffnesses. In fact, the maximum deviations between the actual and the observed variables $\left(0.03 \%\right.$ in $\mathrm{EI}_{4}$ of $\mathrm{FEM}-1.1$ and $0.03 \%$ in $\mathrm{EI}_{5}$ of $\left.\mathrm{FEM}-2.1\right)$ are practically negligible. In models with only three unknown stiffnesses (such as FEM.1.2 and 2.2), the maximum deviations are reduced to $0.01 \%$ and $0.006 \%$, respectively. It is important to highlight, that the accuracy in estimations of these models is significantly higher than that presented by the method proposed by Liao et al. (2012) (1.10\% and 1.94\% in damaged beams 1 and 2, respectively). Furthermore, this accuracy is obtained with a lower number of measurements and without the need of any information from equivalent undamaged structures. A quantitative comparison among methods is presented in Section 4.3.

\subsection{Method 2 [Abdo (2012)]}

According to the method proposed by Abdo, damage in beam structures can be located by analyzing the so 
called grey relation coefficient [Abdo (2012)] of their elements. This coefficient includes changes in displacement curvature between undamaged and damaged structures. Unlike the procedure presented by Liao et al. (2012), this method indicates the damage location but not its magnitude.

\subsubsection{Example 2}

This example is based on two-span continuous beam numerically analyzed by Abdo (2012) and presented in Fig. 5.A. In this structure the damage location of the beam elements is unknown. The FEM of this structure includes 61 nodes and 60 beam elements as showed in Fig. 5.B. The cross sectional area and the inertia of the girder are $0.07 \mathrm{~m}^{2}$ and $0.04 \mathrm{~m}^{4}$, respectively and the mechanical properties are, modulus of elasticity, $E=210$ $\mathrm{GN} / \mathrm{m}^{2}$ and the density, $\rho=7.850 \mathrm{~kg} / \mathrm{m}^{3}$. Damage are assumed to affect only the flexural stiffnesses. This hypothesis leads to 60 unknown parameters.

(A) Geometry

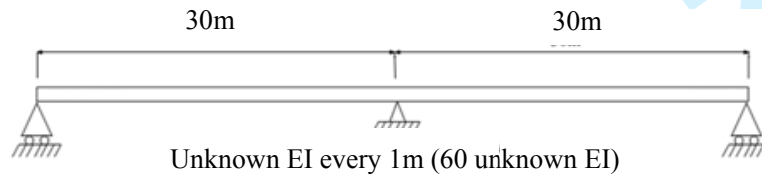

(B) FEM

Fig 5: Example 2: (A) Geometry, and (B) FEM. vertical deflections at inner points of the undamaged and the damaged structure. From these deflections, a Laplacian operator was used to estimate the changes in the analytical and measured displacement curvatures. Analysis of the grey relation coefficient value showed the damage locations. Nevertheless, this analysis did not provide information about their magnitudes.

\subsubsection{Example 2 by observability techniques}

To analyze the Example 2 by observability techniques, an adequate FEM is first defined by the guidelines presented in Section 2. This FEM includes 61 nodes and 60 beam elements with unknown flexural stiffnesses as presented in Fig. 5.B. The load test includes a concentrated load Q of $100 \mathrm{kN}$ at node 16 . The vertical deflections, $\mathrm{v}$, the rotations, $\mathrm{w}$, and the increments of rotations, $\Delta \mathrm{w}$, obtained in test are summarized in Fig. 6.A, 
6.B, and 6.C, respectively. The red circles in Figure 6.C illustrate the location with null increments of rotations.

(A) Vertical deflections, $\mathrm{v}$

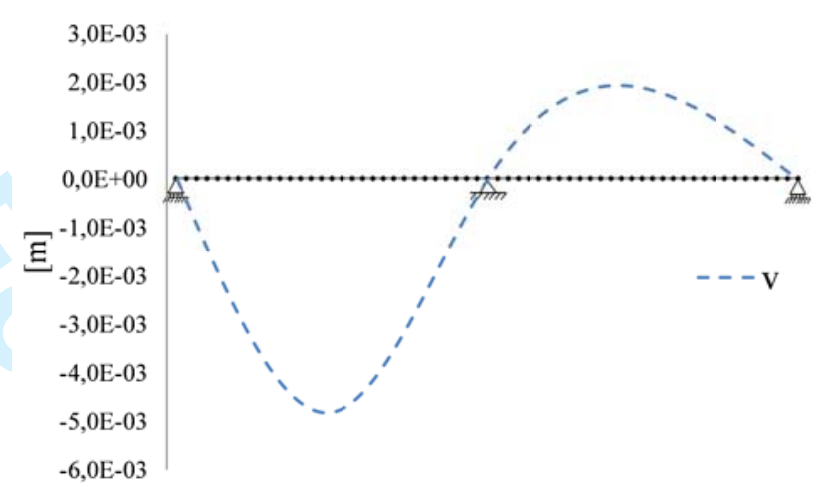

(B) Rotations, w

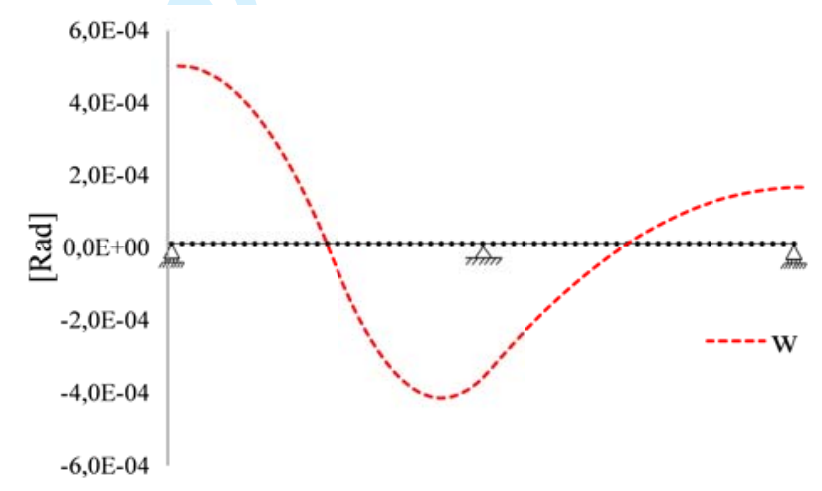

(C) Increments of Rotations, $\Delta \mathrm{w}$

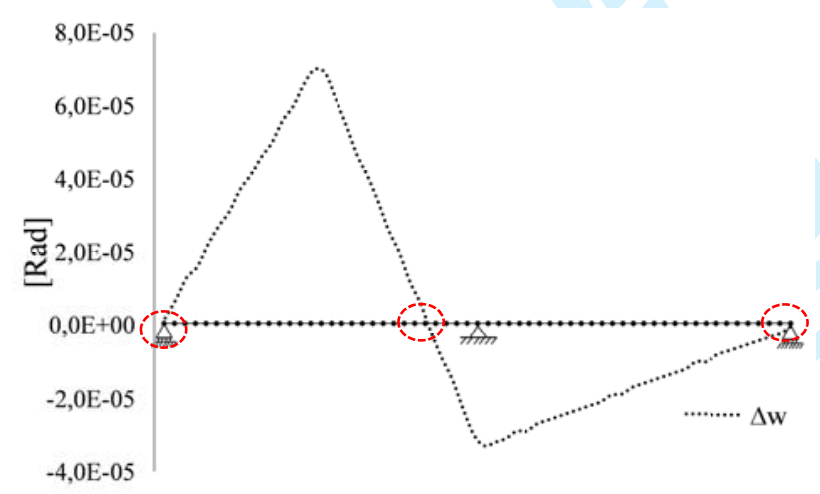

Fig 6: Example 2: Graphical representation of the values introduced in the analysis. The load case corresponds with a concentrated load Q of $100 \mathrm{kN}$ at node 16: (A) Vertical deflections, v, (B) Rotations, w, and (C) Increments of rotations, $\Delta \mathrm{w}$.

For an adequate SSI by observability techniques it is necessary to measure 60 deflections or rotations (this number corresponds with the number of unknown flexural stiffnesses). Two different measurement sets have 
been studied. These sets include 58 common vertical deflections (from $\mathrm{v}_{2}$ to $\mathrm{v}_{29}$ and from $\mathrm{v}_{31}$ to $\mathrm{v}_{60}$ ) and 2 different rotations ( $\mathrm{w}_{29}$ and $\mathrm{w}_{30}$ in the first set and $\mathrm{w}_{30}$ and $\mathrm{w}_{31}$ in the second set). In both sets the flexural stiffnesses are obtained after a recursive process of 60 steps.

In order to illustrate the effect of the errors in the accuracy of the estimations, two different precision in the values obtained in SAP2000 are considered, namely (a) low, 1e-5 m, and (b) high, 1e-9 m. The deviations between the observed and the actual flexural stiffnesses obtained by the two measurement sets for the two precisions are presented in Fig. 7.A and 7.B. The lack of precision might be due to different facts such as the measurement noise. This issue will be addressed in the near future by the authors.

(A) Low precision, 1e-5 m, in measurements

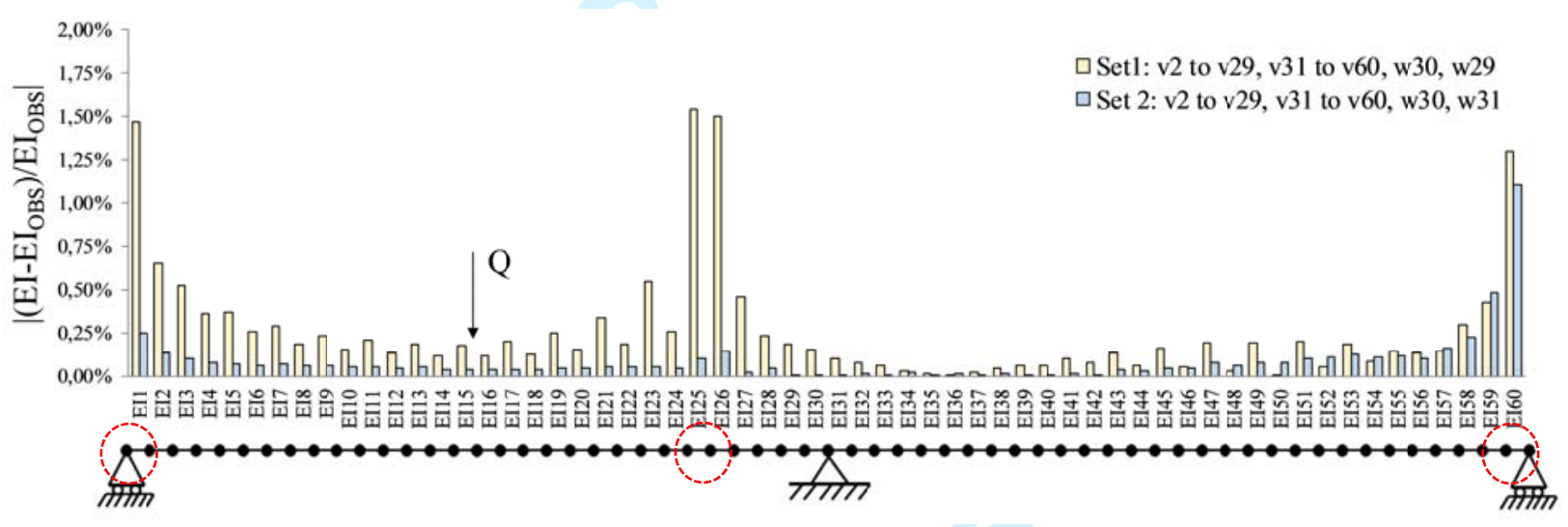

(B) High precision, 1e-9 $\mathrm{m}$, in measurements

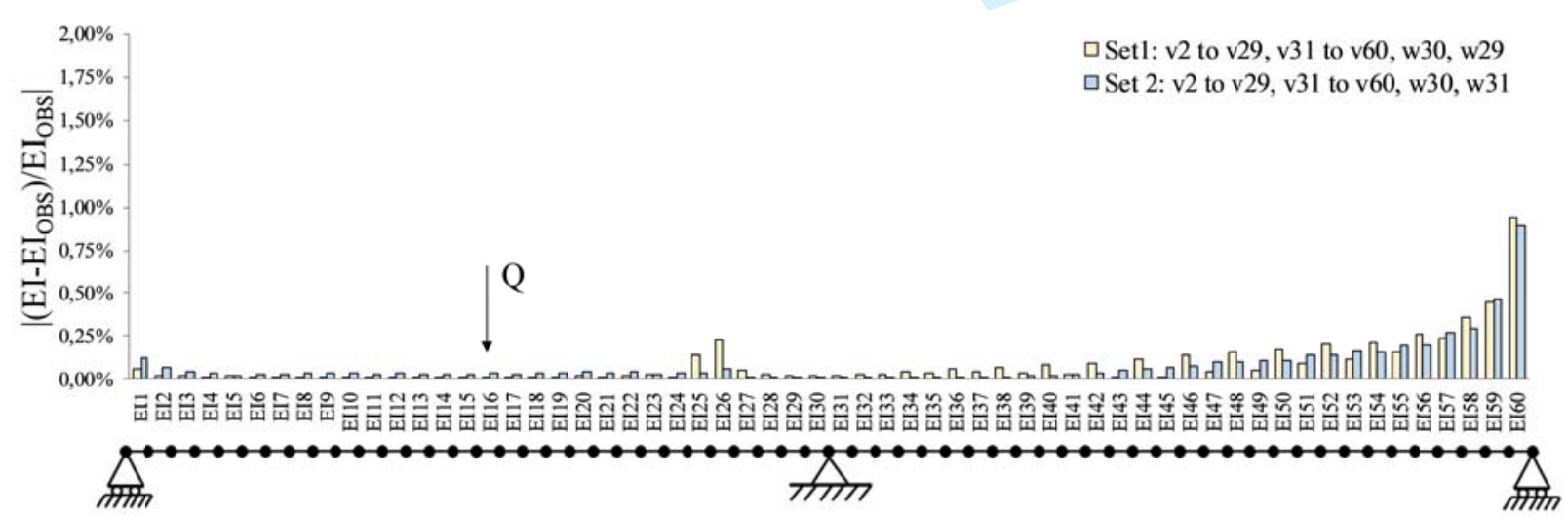

Fig. 7: Deviations between the actual and the observed stiffnesses by two measurement sets in a static test with a concentrated load Q $(100 \mathrm{kN})$ applied at node 16 for two precisions in measurements: (A) Low precision, and (B) High precision. 
Figure 7 shows that the higher the accuracy of the measurements the lower the deviations between the actual and the observed parameters. For example, in the first measurement set the percentage deviations in estimations are reduced from $1.54 \%$ to $0.14 \%$ in $\mathrm{EI}_{25}$ when the accuracy of the measurements is increased. The measurement set also plays an important role in the accuracy of estimations. In fact, Fig. 7 shows that in most locations the second measurement set provides lower deviations than the first measurement set. When the precision of the measurements increases, the higher differences are located at the beam edge $\left(\mathrm{EI}_{60}\right)$. In this element the maximum differences are $0.94 \%$ and $0.89 \%$ for the first and the second measurement set, respectively. This figure also shows that the highest errors are due to three coupled effects. These effects are as follows: (1) Influence of the magnitude of the structural deformation. The effect of the error depends to a great extent on the magnitude of the structural deformation. In fact, it is clear that the higher the static load applied on the structure, the larger the deflections and therefore, the smaller the effect of the measurement errors for a given absolute value. The same effect is produced when the load is located far from the supports as rotations and deflections are higher. For both examples, for a maximum given absolute value of the error, the error percentage for each measurement is lower. From Figure 7.B it can be seen that the estimation error in the first span is lower than in the second one. (2) Influence of the structural deformed shape. In the proposed algorithm, the flexural stiffness of a given element it is mainly estimated from the differences between nodal rotations at its ends. Where the rotation is maximum, the difference between both rotations is minimum (see Figure 6.B and 6.C) and slight numerical errors affect greatly the estimations. This response is highlighted in Figure 7.A with red circles, where maximum errors are found where rotations are maximum and gradient of rotations are minimum. (3) Observability flow: The analysis is performed throughout a recursive process that uses information from preceding steps. For this reason, variables calculated in the latter steps have likely more errors (as they are accumulated). This is appreciated in Figure 7.B.

\subsection{Comparison among methods}

The method proposed by Liao et al. (2012) has several advantages. First of all, damage magnitude can be 
estimated by measuring vertical deflections which can be easily and economically measured. In fact, the application of a moving load makes that instrumentation at only one point (point $M$ in Fig. 2) is required. Furthermore, this method is still applicable when deflections are measured at different locations. For example, to obtain vertical deflections in bridge decks, Ozakgul et al. (2007) proposed the use of tiltmeters and Gentile and Bernardini (2010) proposed the use of radar techniques. Unfortunately, the major disadvantage of this method is the fact that information of the undamaged structure is required. Undamaged structures with similar properties than the analyzed structure can be rarely found on site. Therefore, the applicability of this method is limited to laboratory tests in which properties of the undamaged structure can be obtained. The second disadvantage is the requirement of a large number of measurements to define the influence line. Finally, this method can only update the value of a certain unknown parameter. This is equivalent to quantify a localized damage.

The method proposed by Abdo (2012) can be used for damage location from static measurements. The major disadvantage of this method is that damage is detected from comparison between damaged and undamaged structures. Another disadvantage of this method is that the magnitude of the damage cannot be estimated as it provides information only on the damage location.

The main characteristics of the different analyzed methods are compared in Table 2. These characteristics refer to the requirement of an Iterative (I) or Recursive (R) process, the use of the method for damage Location (L), Quantification $(\mathrm{Q})$ or both $(\mathrm{L}+\mathrm{Q})$, the requirement of information only from the damaged structure (this is, no information of the undamaged structure is required) and the set of measurements used (curvatures, $\chi$, horizontal deflections, u, vertical deflections, v, and/or rotations, w). It is important to highlight that the information introduced into the observability techniques can be obtained with a minimum number of monitored degrees of freedom on site by taking advantage of the Maxwell's reciprocal theorem. This hypothesis has been used in a number of works (see e.g. Strauss et al. (2012) or Degrande and Lombaert (2001)). 
Table 2: Comparison of the main characteristics of the damage detection methods, I/R: Iterative / Recursive Process, L/Q: Locating or Quantifying damage, UN: Undamaged structure.

\begin{tabular}{ccccc}
\hline Method & I/R & L/Q & Data from UN & Measurements \\
\hline Liao et al. (2012) & $\mathrm{I}$ & $\mathrm{Q}$ & $\checkmark$ & $\mathrm{v}$ \\
Abdo (2012) & - & $\mathrm{L}$ & $\checkmark$ & $\mathrm{v}$ to estimate $\chi$ \\
Lozano-Galant et al. $(2013,2014)$ & $\mathrm{R}$ & $\mathrm{L}+\mathrm{Q}$ & $\mathrm{X}$ & $\mathrm{v}$ and/or w \\
\hline
\end{tabular}

The analysis of this table shows that all methods but the one presented by Abdo (2012) use an iterative or recursive process. Observability techniques can be used to both locate and quantify damages. These methods have the additional advantage of not requiring information from the undamaged structure. Referring to the used measurements, the method presented by Liao et al. (2012) is based on v and the method of Abdo (2012) is based on $\chi$. In the case of the observability method $\mathrm{v}$ and/or $\mathrm{w}$ can be introduced.

A comparison between the number of measurements (NM) required by the Existing Methods $\left(\mathrm{NM}_{\mathrm{EM}}\right)$ and the observability technique ( $\mathrm{NM}_{\mathrm{OBS}}$.) in the analyzed examples is presented in Table 3 . This table also includes the Number of Unknown flexural stiffnesses (NU) and the perceptual reduction of the number of measurements required by the observability analysis in comparison with the existing methods. This table includes the information of damaged beams in Examples 1 (FEMs 1.1, 1.2, 2.1 and 2.2) and 2.

Table 3: Comparison of the results of the different examples: NU: Number of Unknown variables, $\mathrm{NM}_{\mathrm{EM}}$ : Number of Measurements in the Existing Method, $\mathrm{NM}_{\mathrm{OBs}}$ : Number of measurements Observability technique. Terms between $/$ refer to FEM with different number of unknown variables.

\begin{tabular}{|c|c|l|l|c|c|}
\hline Example & $\begin{array}{c}\text { Existing method } \\
\mathbf{( E M )}\end{array}$ & $\mathbf{N U}$ & $\mathbf{N M}_{\mathbf{E M}}$ & $\mathbf{N M}_{\mathbf{O B S}}$ & $\begin{array}{c}\mid\left(\mathbf{N M}_{\mathbf{E M}}-\mathbf{N M}_{\mathbf{O B S}} / \mathbf{N M}_{\mathbf{E M}} \mid\right. \\
(\mathbf{\%})\end{array}$ \\
\hline Example 1 (FEMs 1.1 and 2.1) & Liao et al. (2012) & 5 & $(19+19)^{\star}$ & 6 & $84 \%$ \\
\hline Example 1 (FEMs 2.1 and 2.2) & Liao et al. (2012) & 3 & $(19+19)^{\star}$ & 4 & $89 \%$ \\
\hline Example 2 & Abdo. (2012) & 60 & $(58+58)^{\star}$ & 60 & $48 \%$ \\
\hline
\end{tabular}

* The same NM are measured in the damaged and the undamaged structure. This set might not correspond with the minimum one as in the case of the observability technique.

Table 3 shows that the number of required measurements by the observability techniques is significantly lower than the one required by the existing methods. This is especially appreciable in Example 1, where the reduction of required measurements reaches $84 \%$ when the FEMs used by the observability includes five 
unknown flexural stiffnesses (FEM 1.1 and 1.2). This reduction is increased to $89 \%$ when the number of unknown flexural stiffnessses in the FEM is reduced to three (FEM 2.1 and 2.1). Finally, in Example 2, only $48 \%$ of the measurements used by Abdo (2012) are required. It is important to highlight that the values introduced in the column $\mathrm{NM}_{\mathrm{OM}}$ might not correspond with the minimum set as in the case of the observability technique.

A comparison of the Maximum Error in estimated flexural stiffnesses (ME) of the analyzed examples between the Existing Method $\left(\mathrm{ME}_{\mathrm{EM}}\right)$ and the observability technique $\left(\mathrm{ME}_{\mathrm{OBS}}\right)$ is presented in Table 4 . This comparison includes the information of the different damaged beams (Examples 1) and measurement sets (Example 2). In the case of the first two examples only the results of the FEMs with five unknown stiffnesses are presented. This table also includes a column indicating the percentage deviation between the accuracy of both methods.

Table 4: Comparison of the Maximum Errors in estimations (ME): $\mathrm{ME}_{\mathrm{EM}}=$ Maximum Error Existing Method, $\mathrm{ME}_{\mathrm{OBS}}$ : Maximum Error Observability techniques

\begin{tabular}{|c|c|c|c|c|c|c|}
\hline & \multicolumn{2}{|c|}{$\mathrm{ME}_{\mathrm{EM}}(\%)$} & \multicolumn{2}{|c|}{$\mathrm{ME}_{\mathrm{OBS}}(\%)$} & \multicolumn{2}{|c|}{$\mid\left(\mathrm{ME}_{\left.\mathrm{EM}-\mathrm{ME}_{\mathrm{OBS}}\right) / \mathrm{ME}} \mathrm{EM} \mid(\%)\right.$} \\
\hline Example & FEM 1.1 & FEM 2.1 & FEM 1.1 & FEM 2.1 & FEM 1.1 & FEM 2.1 \\
\hline \multirow{3}{*}{ Example 1} & 1.10 & 1.94 & 0.01 & 0.01 & 99.09 & 99.69 \\
\hline & FEM 2.1 & FEM 2.2 & FEM 2.1 & FEM 2.2 & FEM 2.1 & FEM 2.2 \\
\hline & 1.10 & 1.94 & 0.03 & 0.03 & 97.27 & 98.45 \\
\hline
\end{tabular}

\begin{tabular}{|c|c|c|c|c|c|c|}
\hline Example & Set 1 & Set 2 & Set & Set 2 & Set 1 & Set 2 \\
\hline Example 2 & - & - & 0.94 & 0.89 & - & - \\
\hline
\end{tabular}

Table 4 shows that the accuracy of the observability method cannot be compared with the one of the existing method in Example 2 because the existing method does not provide the estimation of the damage extent. Therefore, the comparison of the accuracy in estimations between methods can only be performed in Example 1 . In this case, the increase of accuracy when observability techniques are used is significant. For example, the maximum errors when observability techniques are used are obtained in FEM 1.1. The value of this error, $0.01 \%$, is practically negligible. 


\section{CONCLUSIONS}

This paper proposes the first numerical application of observability techniques to damage detection of structures from static monitoring information. With this aim, a symbolical-numerical algorithm, named Numerical Observability Method is developed to deal with the numerical complexities derived from the application of observability techniques. This algorithm is complemented with an algorithm addressing the definition of effective finite element models.

The observability technique has been validated by means of a comparison with two alternative static-excitation based methods proposed in the literature. In all analyzed examples the number of deflections required by the observability techniques is significantly lower than those required by alternative methods. The major advantage of the observability techniques in comparison with the other analyzed methods is that no data of the undamaged structure is required. All these characteristics show the convenience of applying the observability techniques for damage detection in bridges. The numerical analysis carried out in this paper shows the important role of the measurement errors. This issue will be studied in detail in the near future by the authors.

\section{ACKNOWLEDGEMENT}

The authors are indebted to the Secretary of State of the Spanish Ministry of Science and Innovation (Project TRA2010-17818, BIA2009-10483 and BIA2009-13056), the Spanish Ministry of Economy and Competitiveness (BIA2013-47290-R) and to the Junta de Comunidades de Castilla-La Mancha (Project PII2I090129-4085), for partial support of this work.

\section{REFERENCES (4/28)}

1. Abdo, M.A. (2012). Parametric study of using only static response in structural damage detection. Engineering Structures, 34, 124-131.

2. ASCE. (2011). Structural identification (st-id) of constructed facilities: approaches, methods and technologies for effective practice of st-id. Technical report, ASCE SEI Committee on Structural Identification of Constructed Systems, American Society of Civil Engineers (ASCE). 
3. Bakhtiari-Nejad, F., Rahai, A., and Esfandiari, A. (2005). A structural damage detection method using static noisy data. Engineering Structures, 27(12):1784-1793.

4. Banan M.R. and Hjelmstad K.D. (1993). Identification of structural systems from measured responses. Technical report, Report No. SRS 579, UILU-ENG 93-2002, Univ. of Illinois at Urbana-Champaign, Urbana, Illinois.

5. Banan, M.R., Banam, M.R. and Hjelmstad, K.D. (1994a). Parameter estimation of structures from static response I: computational aspects. Journal Structural Engineering 120, 3243-3258.

6. Banan, M.R., Banam, M.R. and Hjelmstad. K.D. (1994b). Parameter estimation of structures from static response II: numerical simulation. Journal Structural Engineering 120, 3259-3283.

7. Castillo, E., Lozano-Galant, J.A., Nogal, M. and Turmo. J. (2014). New tool to help decission making in civil engineering. Journal of Civil Engineering and Management, In press.

8. Chao, S.H and Loh, C.H. (2014) Application of singular spectrum analysis to structural monitoring and damage diagnosis of bridges, Structure and Intrastructure Engineering, 10(6), 708-727.

9. Chang, K.C., Kim, C.W. and Kawatani, M. (2014) Feasibility investigation for a bridge damage identification method through moving vehicle laboratory experiment, Structure and Intrastructure Engineering, 10(3), 328-345.

10. Degrande, G. and Lombaert, G. (2001) An efficient formulation of Krylov's prediction model for train induced vibrations based on the dynamic reciprocity theorem, Acoustical Society of America, 110(3), 1379-1389.

11. Gentile C. and Saisi, A. (2011). Ambient vibration testing and condition assessment of the paderno iron arch bridge (1889). Engineering Structures, 25(9):3709-3720.

12. Hjelmstad, K.D., Wood. S.L., and Clark. S.J. (1992). Mutual residual energy method for parameter estimation in structures. ASCE Journal of Structural Engineering, 118(1):223-242.

13. Hjelmstad K.D. and Shin. S. (1997). Damage detection and assessment of structures from static response. Journal of Engineering Mechanics, 123(6):586-576.

14. Liao, J., Tang, G. Meng, L., Liu, H., andZhang, Y. (2012). Finite Element Model Updating Based on Field Quasi-static Generalized Influence Line and Its Bridge Engineering Applications. Procedia Engineering 31, 348-353.

15. Liu P.L. and Chian. C.C. (1997). Parametric identification of truss structures using static strains. Journal of Structural Engineering, 123(7), 927-933.

16. Lozano-Galant, J.A., Nogal, M., Castillo, E. and Turmo, J. (2013). Application of Observability Techniques to Structural-System Identification. Computer Aided-Civil and Infrarestructure Engineering, 28(6), 434-454. 
17. Lozano-Galant, J.A., Nogal, M., Payá-Zaforteza, I. and Turmo, J. (2014). Structural System Identification of Cable-Stayed Bridges with Observability Techniques, Structure and Intrastructure Engineering, 10(11), 1331-1344.

18. Lozano-Galant, J.A. Nogal, M. Turmo, J. Castillo, E. (2015) Selection of Measurments Sets in Static Structural Identification of Bridges using Observability Trees, Computers and Concrete, An Inernational Journal, 15(3).

19. Ozakgul, K., Caglayan, O. and Uzgider, E. (2007). Load testing of bridges using tiltmeters. In Proceedings of the SEM Annual Conference, Albuquerque New Mexico USA, June 1-4.

20. Rezaiee-Pajand, M. Kazemiyan, M.S. and Aftabi. A. (2014) Static Damage Identification of 3D and 2D Frames, Mechanics Based Design of Structures and Machines: An International Journal, 42 (1), 70-96.

21. Remon, A., Quintana-Ortí, E.S., Quintana-Ortí, G. (2006) Parallel LU Factorization of Band Matrices on SMP Systems, High Performance Computing and Communications, Lecture Notes in Computer Science, 4208, 110-118.

22. Sanayei M. and Scampoli, S.F. (1991). Structural element stiffness identification from static test data. Journal of Engineering Mechanics, 117(5):1021-1036.

23. Sanayei, M. and Onipede, O. (1991). Damage assessment of structures using static test data. $A I A A$ Journal, 29(7):1174-1179.

24. Sanayei, M., Onipede, O. and Babu. S.R. (1992). Selection of noisy measurement locations for error reduction in static parameter identification. AIAA Journal, 30(9):2299-2309.

25. Sanayei M. and Saletnik M.J. (1996a). Parameter estimation of structures from static strain measurements I: Formulation. Journal of Structural Engineering, 122(5):555-562.

26. Sanayei M. and Saletnik M.J. (1996b). Parameter estimation of structures from static strain measurements II: Error sensitivity analysis. Journal of Structural Engineering, 122(5):563-572.

27. Sanayei, M., Phelps, J.E., Sipple, J.D. and Bell, B.R. and Brenner E.S. (2012). Instrumentation, nondestructive testing, and finite-element model updating for bridge evaluation using strain measurements. Journal of Bridge Engineering, 17(1):130-138.

28. Strauss, A. Wendner, R., Frangopol, D.M. and Bergmeister, K. (2012). Influence line-model correction approach for the assessment of engineering structures using novel monitoring techniques, Smart Structures and Systems, 9(1), 1-20.

29. Viola, E. and Bocchini, P. (2013). Non-destructive parametric system identification and damage detection in truss structures by static tests. Structure and Infrastructure Engineering, 9(5), 384-402.

30. Yang, H. and Zhang, P.P. (2014) A note on multiplicative perturbation bounds for the MoorePenrose inverse, Linear \& Multilinear Algebra, 62(6), 831-838. 
31. Zhang, J, Hong, W., Tang, Y.S., Yang, C.Q., Wu, G. and Wu, Z.S. (2014) Structural health monitoring of a steel stringer bridge with area sensing, Structure and Intrastructure Engineering, 10(8), 1049-1058. 